\title{
3-Methylglutaconic aciduria type III in a non-Iraqi-Jewish kindred: clinical and molecular findings
}

\author{
Robert Kleta, ${ }^{\text {a }}$ Flemming Skovby, ${ }^{\mathrm{b}}$ Ernst Christensen, ${ }^{\mathrm{b}}$ Thomas Rosenberg, ${ }^{\mathrm{c}}$ \\ William A. Gahl, ${ }^{\mathrm{a}, *}$ and Yair Anikster ${ }^{\mathrm{a}, 1}$ \\ a Section on Human Biochemical Genetics, Heritable Disorders Branch, National Institute of Child Health and Human Development, \\ National Institutes of Health, 10 Center Drive, MSC 1830, Building 10, Room 9S-241, Bethesda, MD 20892-1830, USA \\ ${ }^{\mathrm{b}}$ Department of Clinical Genetics, Copenhagen University Hospital, Copenhagen, Denmark \\ ${ }^{\mathrm{c}}$ Gordon Norrie Centre for Genetic Eye Diseases, National Eye Clinic for the Visually Impaired, Hellerup, Denmark
}

Received 9 April 2002; received in revised form 30 April 2002; accepted 10 May 2002

\begin{abstract}
Type III 3-methylglutaconic aciduria (MGA) (MIM 258501) consists of early bilateral optic atrophy, later development of spasticity, extrapyramidal dysfunction and occasionally cognitive deficits, and urinary excretion of 3-methylglutaconic acid and 3methylglutaric acid. The presence of the disorder in an Iraqi-Jewish genetic isolate led to mapping of the OPA3 gene to chromosome 19q13.2-q13.3, followed by isolation of the gene itself. OPA3 consists of two exons and codes for a peptide of 179 amino acids. Iraqi-Jewish patients with type III MGA are homozygous for a splice site founder mutation in OPA3 (IVS1-1G > C) which abolishes mRNA expression in fibroblasts. Here we report a novel mutation in OPA3 (320-337del) in a Kurdish-Turkish patient with optic atrophy and 3-methylglutaconic and 3-methylglutaric aciduria, previously carrying the diagnosis of type IV MGA. We conclude that type III MGA occurs in patients of non-Iraqi-Jewish ancestry, and should be considered in patients with type IV MGA that have optic atrophy and ataxia. (C) 2002 Elsevier Science (USA). All rights reserved.
\end{abstract}

Keywords: 3-Methylglutaconic acid; 3-Methylglutaric acid; Optic atrophy; Ataxia; Mitochondrion

\section{Introduction}

Based upon clinical and laboratory findings, 3methylglutaconic aciduria (MGA) has been divided into four categories [1]. Type I MGA manifests with mild neurological disease; the basic defect involves deficiency of 3-methylglutaconyl-CoA hydratase, an enzyme in the leucine oxidation pathway. Type II MGA, also called Barth syndrome, consists of dilated cardiomyopathy, short stature and neutropenia, and results from mutations in the X-linked tafazzin (G4.5) gene [2]. Type III MGA, or Costeff optic atrophy syndrome, has been described only in a genetic isolate of Iraqi Jews, and manifests with neurophthalmic symptoms. Finally, type

\footnotetext{
${ }^{*}$ Corresponding author. Fax: +301-402-2740.

E-mail address: bgahl@helix.nih.gov (W.A. Gahl).

${ }^{1}$ Present address: Metabolic Unit, Sheba Medical Center, Tel Hashomer 52621, Israel.
}

IV MGA presents with variable neurologic disease and occasional cardiac, ophthalmic, hepatic, and renal symptoms.

Type III MGA (MIM 258501) was mapped by linkage analysis to chromosome 19q13.2-q13.3 [3] and the causative gene, $O P A 3$, was recently isolated. A founder mutation was subsequently identified among Iraqi-Jewish individuals with type III MGA [4]. These patients exhibit early bilateral optic atrophy, spasticity, extrapyramidal dysfunction, ataxia, and cognitive deficits [5,6], but normal longevity [7]. 3-Methylglutaconic acid and 3-methylglutaric acid concentrations are elevated in the urine, so the diagnosis can be made as early as 1 year of age in infants with optic atrophy. Here we report the $O P A 3$ mutation in a Kurdish-Turkish patient previously diagnosed with type IV MGA. We suggest that the diagnosis of type III MGA should be entertained in patients with optic atrophy and 3-methylglutaconic aciduria, even if they are not of Iraqi-Jewish ancestry. 


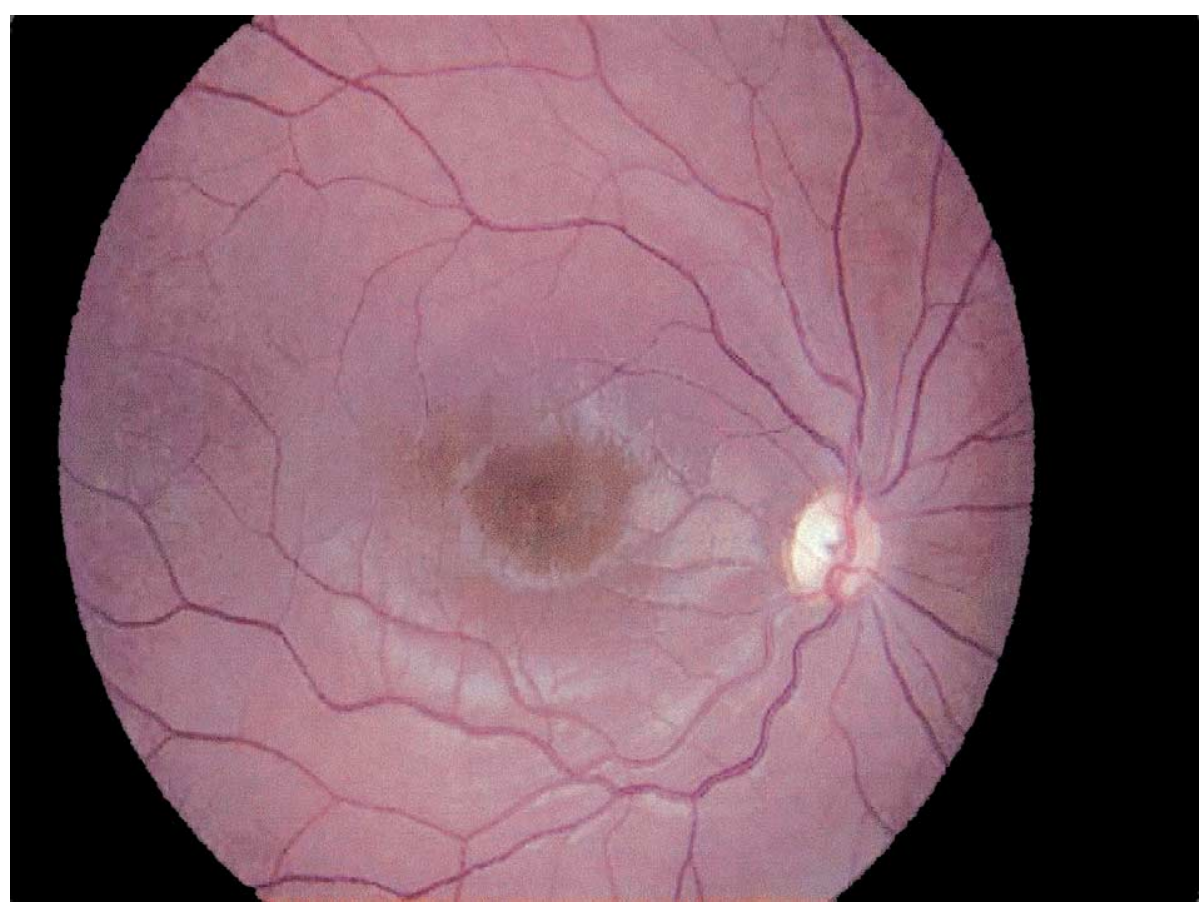

Fig. 1. Fundus photograph of the right eye showing temporal pallor of the optic disc. The brown pigment in the macula region represents a normal finding in a young dark-haired individual.

A

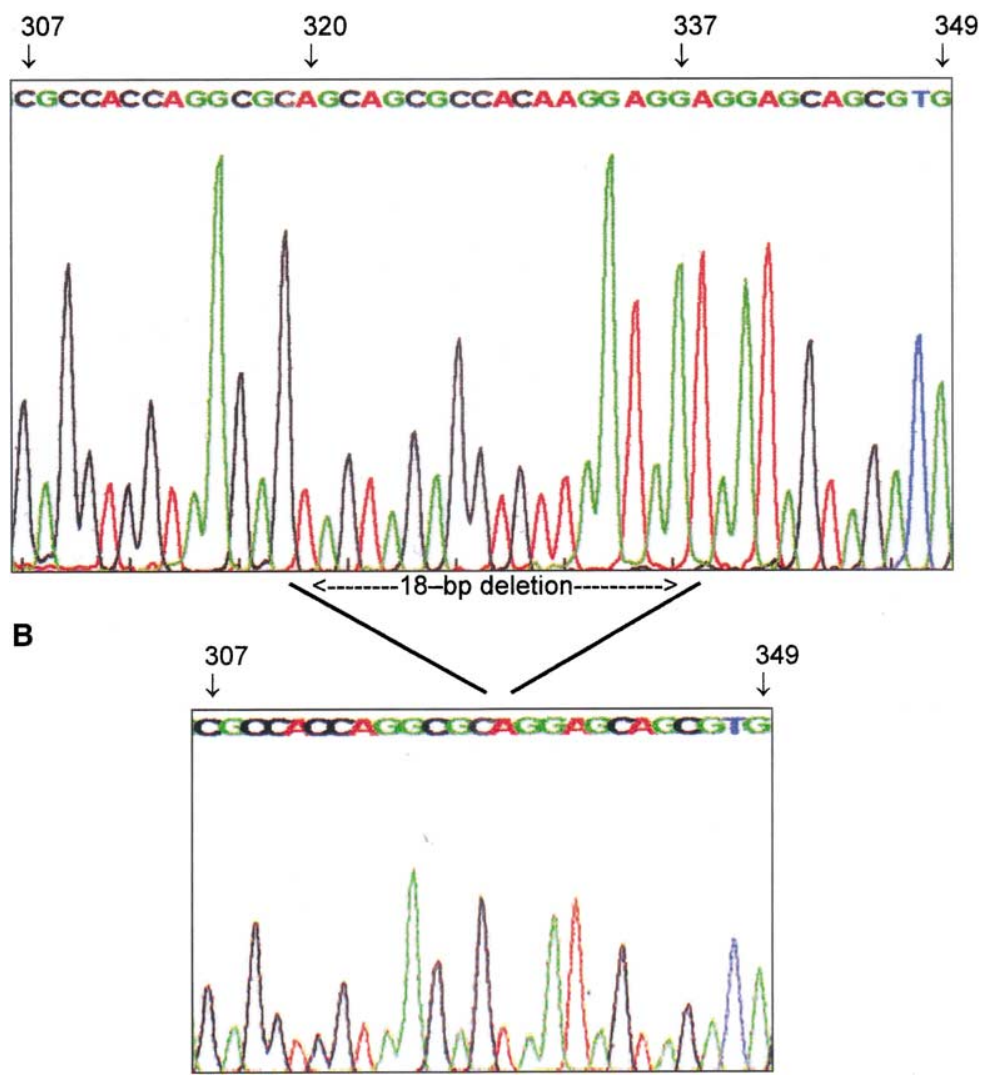

Fig. 4. Region of exon 2 of $O P A 3$ showing control (A) and patient (B) sequence. The patient exhibited a deletion of nucleotides 320-337. Numbers refer to amino acid positions, with 1 referring to the ATG start codon. 
A

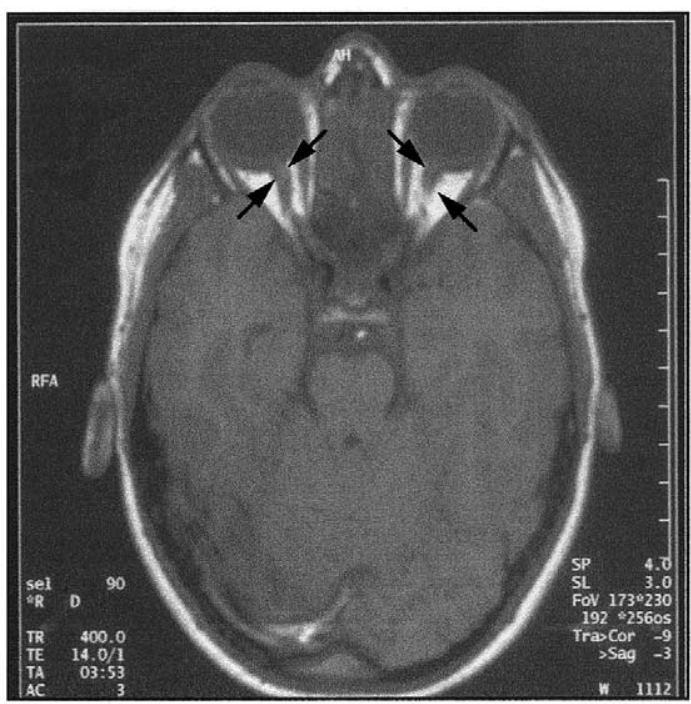

C

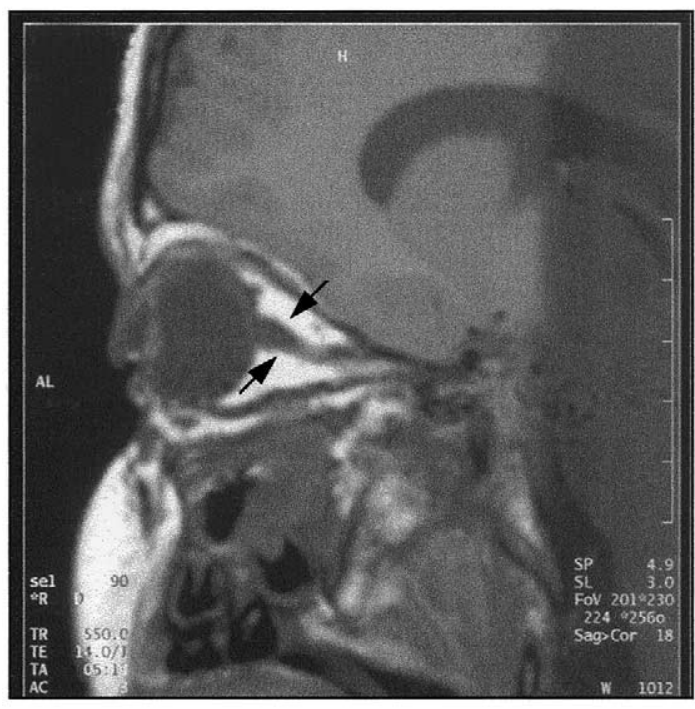

B

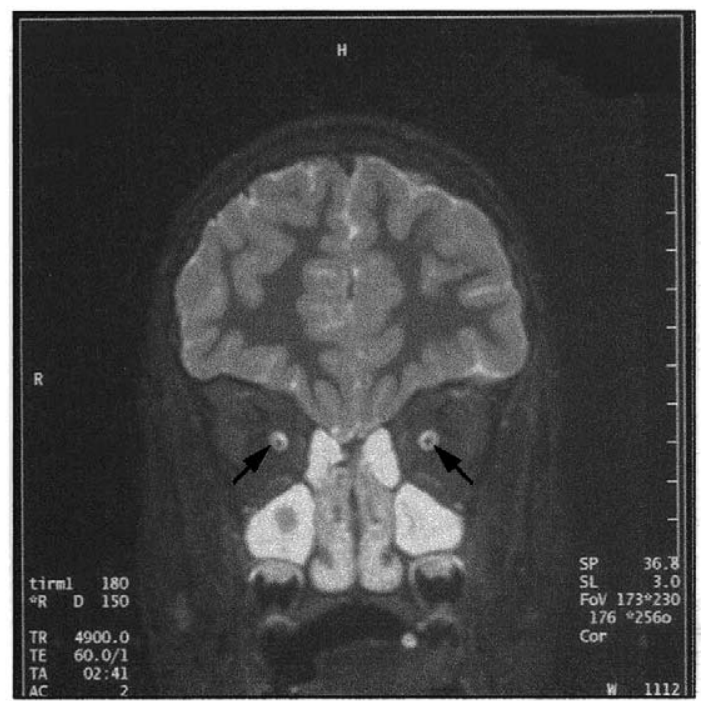

D

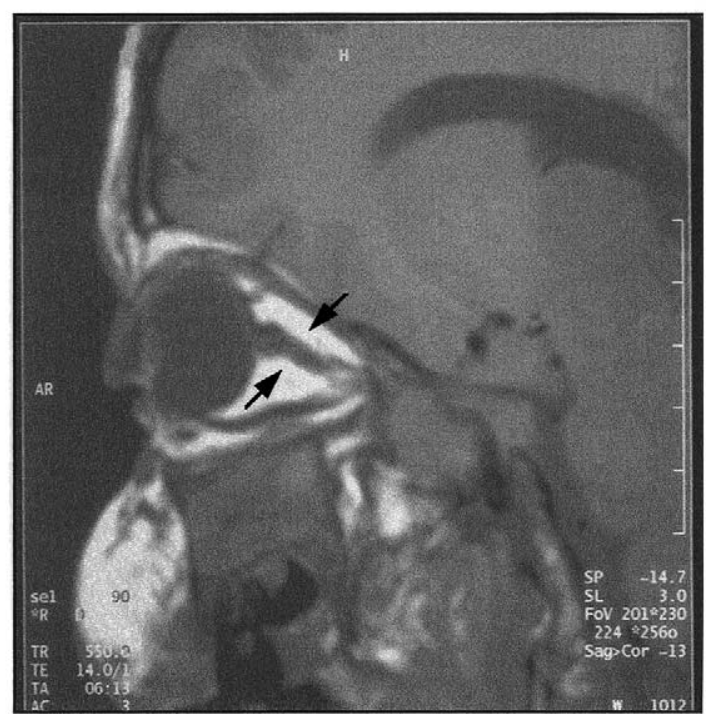

Fig. 2. Brain MRI showing atrophy of the optic nerves (indicated by arrows). (A) Horizontal T1-weighted. (B) Coronal T2-weighted. (C) Sagittal T1weighted right eye. (D) Sagittal T1-weighted left eye.

\section{Patient and methods}

\subsection{Case report}

The patient is a 14 year old boy whose KurdishTurkish parents are first cousins. An older brother and a younger sister are both healthy. The patient was born at term after an uncomplicated pregnancy and delivered by C-section because of breech presentation. Birth weight was $3400 \mathrm{~g}$, length $53 \mathrm{~cm}$, and no dysmorphology was ever noted. The postnatal course was uncomplicated.

The patient walked at 11 months but the gait was unsteady at 16 months. CT of the brain was normal.
Organic acid analysis of the urine revealed elevated 3methylglutaconic acid and 3-methylglutaric acid. An assay on cultured fibroblasts for 3-methylglutaconylcoenzyme A hydratase, kindly performed by K.M. Gibson, Ph.D. (Biochemical Genetics Laboratory, Portland, Oregon, USA), was normal. At 19 months, mental development was appropriate for age, but the gait was ataxic and broad-based. Biotin $(1 \mathrm{mg}$, p.o., t.i.d.), riboflavin (100 $\mathrm{mg}$, p.o., t.i.d.), and protein restriction had no effect on the organic aciduria or ataxia.

Major motor milestones were reached in spite of nonprogressive ataxia, mild hypotonia, and hypermobility. The boy played soccer and other sports, and did well in 


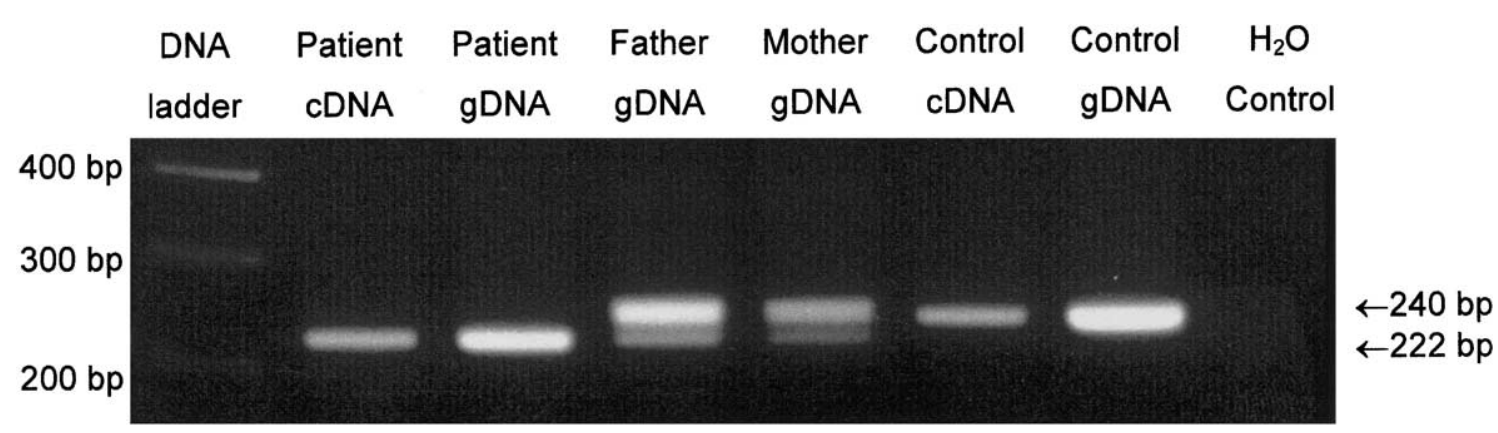

Fig. 3. Gel electrophoresis of the PCR amplified products of exon 2 of $O P A 3$ using cDNA and gDNA from the patient, gDNA from his parents, and both cDNA and gDNA controls. Control and parental DNA yielded the expected 240-bp fragment. The patient's DNA, as well as his parents', produced a smaller band indicating a deletion within the amplified region.

kindergarten. Poor concentration and motor restlessness were noted in first grade, and absence seizures were suspected. An electroencephalogram, normal at age 2 years, now revealed $3-4 \mathrm{~Hz}$ spike foci in both occipital lobes. Oxcarbazepin was administered but discontinued because of increased ataxia. An ophthalmologic evaluation at age 9 showed decreased visual acuity bilaterally (6/18). Fundi were pale temporally and showed optic atrophy (Fig. 1). Visual evoked potentials had a broad configuration with normal latency and amplitude. A brain magnetic resonance image (MRI) revealed atrophy of the optic nerves, in both their retrobulbar and intracranial pathways (Fig. 2). An electroretinogram was not performed. Acousticovestibular examination was normal at the age of 11 years. When last examined at the age of 13 years, visual acuity was moderately reduced (6/36) with a pronounced crowding phenomenon.

Linear growth was between the 10th and 25th percentiles. The patient performs well in mathematics and adequately in other subjects. He is physically active. The most recent neurologic examination at age 13 years was unremarkable except for constant, mild ataxic restlessness. There was no cardiomyopathy, and the peripheral blood smear was normal.

\section{Sequencing}

After informed consent, genomic DNA (gDNA) was extracted from whole blood using a standard method [8], and a skin biopsy was also performed. In addition, total RNA was extracted from cultured fibroblasts using Trizol reagent (Life Technologies). cDNA was synthesized by reverse transcription using $5 \mu \mathrm{g}$ of total RNA and a SuperScript First-Strand Synthesis System for RT-PCR kit, according to manufacturer's protocol (GIBCO-BRL).

Amplification of each exon of the $O P A 3$ gene was performed using 200-400 ng of gDNA, Ready-To-Go PCR Beads (Amersham Pharmacia Biotech), and $10 \mathrm{pmol}$ of each primer in a total volume of $25 \mu \mathrm{L}$, as described in [4]. The initial denaturation step consisted of $96^{\circ} \mathrm{C}$ for $4 \mathrm{~min}$, followed by 35 cycles of denaturation at $96^{\circ} \mathrm{C}$ for $30 \mathrm{~s}$, annealing at $60^{\circ} \mathrm{C}$ for $30 \mathrm{~s}$, and extension at $72^{\circ} \mathrm{C}$ for $30 \mathrm{~s}$, concluding with an elongation step at $72^{\circ} \mathrm{C}$ for $10 \mathrm{~min}$. Electrophoresis of the products was performed using $1 \%$ agarose. We sequenced the $O P A 3$ coding exons and their adjacent splice site regions using gDNA of the affected individual and his parents. Primers were as follows: F1: 5'-CGTACATACGT ACTGACGCA-3'; R1: 5'-TAAGCAACCACCTGACA GG-3'; F2: 5'-TCCCAGAGCGCAGCCTGAC-3'; R2: $5^{\prime}$-GCCAAGTTGCATCAAGATCCT- $3^{\prime}$. To further characterize the deletion in exon 2 , we used primers Del-F, 5'-GAAGCCACCATCTTCATCGTGGG-3', and Del-R, 5'-ATTGCAGAGCTGGGCGCGCACCT$3^{\prime}$. For automated sequencing, we employed a Beckman CEQ 2000XL sequencer and CEQ Dye Terminator Cycle Sequencing kit according to manufacturer's protocol (Beckman Coulter). BLAST analyses were performed using searches provided by the National Center for Biotechnology Information (http://www.ncbi.nlm. nih.gov/BLAST/).

\section{Results}

We first investigated our patient's cDNA for the presence of the Iraqi-Jewish OPA3 mutation (IVS1$1 \mathrm{G}>\mathrm{C}$ ), a homozygous $\mathrm{G}-\mathrm{C}$ change at the -1 position of intron 1 in the $3^{\prime}$ (acceptor) splice site. This mutation abolishes $O P A 3$ mRNA expression. Using the patient's cDNA as template, we were able to amplify the $O P A 3$ gene, indicating that this boy did not have the founder Iraqi-Jewish mutation. Rather, PCR amplification of exon 2 of $O P A 3$ using the patient's gDNA revealed a slightly smaller fragment compared with that produced using his parents' DNA. On high percentage agarose gel electrophoresis, a lower band was present for the patient and two bands were seen for each parent, indicating homozygosity and heterozygosity, respectively, for a small deletion (Fig. 3). Sequencing revealed an in-frame deletion of nucleotides AGCAGCGCCACAAGGAGG 
at positions 320-337 in exon 2 (Fig. 4), resulting in deletion of the six amino acids between residues 108 and 113, i.e., QRHKEE.

\section{Discussion}

Several metabolic diseases are characterized by the urinary excretion of 3-methylglutaconic and 3-methylglutaric acids, intermediates in leucine metabolism and the mevalonate shunt pathway involving isoprenoid or sterol biosynthesis. These disorders, especially type IV MGA, have been difficult to categorize for years. Here we present the first evidence that at least one type IV MGA patient really has type III MGA. In addition, the finding of a unique $O P A 3$ mutation in a KurdishTurkish kindred indicates that type III MGA occurs outside of the Iraqi-Jewish population. In that genetic isolate, the complete absence of an $O P A 3$ transcript causes early onset optic atrophy and a movement disorder of variable severity, beginning in adolescence. We now show that a milder mutation in $O P A 3$ may also cause optic atrophy early in life.

The function of the $O P A 3$ gene product remains unknown, but a targeting motif in the sequence suggests a role in the mitochondrion [4]. Our patient's symptoms also included ataxia, which might reflect mitochondrial involvement of the central nervous system [9]. Mitochondrial disorders, including respiratory chain defects, can affect high-energy consuming structures such as the basal ganglia and the cerebellum, causing acute or chronic ataxia. Mitochondrial defects can also present with optic atrophy. A dominant form of optic atrophy (Kjer type) is the most common inherited optic neuropathy, and the responsible gene, OPA1, encodes a mitochondrial dynamin-related GTPase [10]. Leber's hereditary optic neuropathy constitutes another example of mitochondrial involvement in a disorder manifesting optic atrophy [11].

The phenotype combining ataxia and optic atrophy resembles the clinical picture described by Carl Behr in 1909 as "complicated infantile familial optic atrophy" (komplizierte infantile familiäre Optikusatrophie) [12], later referred to as Behr disease [13]. Behr described six male children aged 3-13 years with optic atrophy and mild ataxia as well as mild spasticity and mental deficiency. His patients constitute a bridge between the hereditary optic atrophies and the ataxias. Behr wrote, "the etiology of this familial disease will remain unknown as long as our knowledge of developmental biochemistry is so rudimentary." The molecular findings in our young patient with type III MGA and clinical manifestations of $\mathrm{Behr}$ disease contribute to a meaningful categorization of both the methylglutaconic acidurias and the hereditary ataxias [14].
In summary, we present evidence of a second $O P A 3$ mutation causing type III MGA and a clinical phenotype involving both optic atrophy and ataxia. Patients with 3-methylglutaconic and 3-methylglutaric aciduria, as well as those with early onset optic atrophy and ataxia, should be screened for mutations in $O P A 3$ to determine the full spectrum of this disorder.

\section{Electronic-database information}

Accession number and URLs for data in this article are as follows:

GenBank, http://www.ncbi.nlm.nih.gov/Web/Genbank/index.html (for Accession Number GI: 13376716 for Homo sapiens optic atrophy 3 (autosomal recessive, with chorea and spastic paraplegia) mRNA (OPA3)).

Online Mendelian Inheritance in Man (OMIM), http://www.ncbi.nlm.nih.gov/Omim/ (for 3-methylglutaconic aciduria type III [MIM 258501]).

\section{Acknowledgments}

The authors thank Dr. K.M. Gibson for performing the fibroblast assay for 3-methylglutaconyl-coenzyme A hydratase. Y.A. is a Howard Hughes Medical Institute Physician Postdoctoral Fellow.

\section{References}

[1] K.M. Gibson, O.N. Elpeleg, C. Jakobs, H. Costeff, R.I. Kelley, Multiple syndromes of 3-methylglutaconic aciduria, Pediatr. Neurol. 9 (1993) 120-123.

[2] S. Bione, P. D'Adamo, E. Maestrini, A.K. Gedeon, P.-A. Bolhuis, D. Toniolo, A novel X-linked gene, G4.5. is responsible for Barth syndrome, Nat. Genet. 12 (1996) 385-389.

[3] A. Nystuen, H. Costeff, O.N. Elpeleg, N. Apter, B. Bonne-Tamir, H. Mohrenweiser, N. Haider, E.M. Stone, V.C. Sheffield, IraqiJewish kindreds with optic atrophy plus (3-methylglutaconic aciduria type 3) demonstrate linkage disequilibrium with the CTG repeat in the $3^{\prime}$ untranslated region of the myotonic dystrophy protein kinase gene, Hum. Mol. Genet. 6 (1997) 563569.

[4] Y. Anikster, R. Kleta, A. Shaag, W.A. Gahl, O. Elpeleg, Type III 3-methylglutaconic aciduria (optic atrophy plus syndrome, or Costeff optic atrophy syndrome): identification of the $O P A 3$ gene and its founder mutation in Iraqi Jews, Am. J. Hum. Genet. 69 (2001) 1218-1224.

[5] H. Costeff, N. Gadoth, N. Apter, M. Prialnic, H. Savir, A familial syndrome of infantile optic atrophy, movement disorder, and spastic paraplegia, Neurology 39 (1989) 595-597.

[6] H. Costeff, O. Elpeleg, N. Apter, P. Divry, N. Gadoth, 3Methylglutaconic aciduria in "Optic Atrophy Plus", Ann. Neurol. 33 (1989) 103-104.

[7] O.N. Elpeleg, H. Costeff, A. Joseph, I. Shental, R. Weitz, K.M. Gibson, 3-Methylglutaconic aciduria in the Iraqi-Jewish 'optic atrophy plus' (Costeff) syndrome, Dev. Med. Child Neurol. 36 (1994) 167-172. 
[8] J. Sambrook, E.F. Fritsch, T. Maniatis, Molecular Cloning: A Laboratory Manual, second ed., Cold Spring Harbor Laboratory, Cold Spring Harbor, NY, 1989.

[9] J. Kaplan, Spinocerebellar ataxias due to mitochondrial defects, Neurochem. Int. 40 (2002) 553-557.

[10] C. Delettre, G. Lenaers, L. Pelloquin, P. Belenguer, C.P. Hamel, OPA1 (Kjer type) dominant optic atrophy: a novel mitochondrial disease, Mol. Genet. Metab. 75 (2002) 97-107.

[11] V. Carelli, F.N. Ross-Cisneros, A.A. Sadun, Optic nerve degeneration and mitochondrial dysfunction: Genetic and acquired optic neuropathies, Neurochem. Int. 40 (2002) 573-584.
[12] C. Behr, Die komplizierte, hereditär-familiäre Optikusatrophie des Kindesalters. Ein bisher nicht beschriebener Symptomkomplex, Klin. Mbl. Augenheilkunde 47 (1909) 138-160.

[13] H.E. Henkes, A.F. Deutman, H.F.M. Busch, Behr disease, in: P.J. Vinken, G.W. Bruyn (Eds.), Handbook of Clinical Neurology, vol. 13, North-Holland Publishing Company, Amsterdam, 1972, pp. 88-93, Chapter 4.

[14] R.N. Sheffer, J. Zlotogora, O.N. Elpeleg, J. Raz, D. Ben-Ezra, Behr's syndrome and 3-methylglutaconic aciduria, Am. J. Ophthalmol. 114 (1992) 494-497. 